\title{
Evaluation of malting quality of spring barley genetic resources from different regions of origin
}

\author{
Marta Zavřelová ${ }^{*}$, Vratislav Psota ${ }^{2}$, Pavel Matušinsky ${ }^{1}$, Markéta Musilová $^{2}$, Michaela Némethová $^{3}$ \\ 1 Agrotest fyto Ltd., Havličkova 2787/121, \\ CZ 76701 Kroměřiž, Czech Republic \\ 2 Research Institute of Brewing and Malting, \\ Mostecká 971/7, CZ 61400 Brno, Czech Republic \\ 3 Mendel University in Brno, Department of Food Technology (FA), \\ Zemědělská 1, CZ 61300 Brno, Czech Republic \\ *Corresponding author: zavrelova@vukrom.cz
}

\begin{abstract}
A set of 92 genetic resources of spring barley split into groups according to the areas of origin was studied in terms of grain malting quality. The following malting parameters were monitored in this study: nitrogen content in barley grain, malt extract, relative extract at $45^{\circ} \mathrm{C}$, Kolbach index, diastatic power, apparent final attenuation, friability, $\beta$-glucans in wort, haze of wort measured at the angles of $15^{\circ}$ and $90^{\circ}$. In the studied set, the largest variability was found in the haze of wort $\left(90^{\circ}\right)$, the smallest in the apparent final attenuation. The optimal values of the extract content in the malt dry matter according to the malting quality index were only detected in the genotypes originating from Europe. The group of the genetic resources from South America and Australia, together with the genotypes from the Middle East and Africa, differed statistically significantly in the values of the Kolbach index from other groups. The two-row genotypes differed statistically significantly in the values of the malt extract content, relative extract at $45^{\circ} \mathrm{C}$, Kolbach index, diastatic power, friability and $\beta$-glucan content in wort. Although the European and non-European groups differed in a number of traits, the non-European groups also included genetic resources that in their malting parameters approached or equaled the European malting varieties (such as Psaknon). In this study, several significant correlations were found; the strongest correlations were between friability and malt extract $(r=0.85)$, friability and Kolbach index $(r=0.84)$.
\end{abstract}

Key words: Hordeum vulgare L., genetic resources, malting quality

\section{Introduction}

Barley is one of the world's most important crops. Breeding and growing of malting barley has a long tradition in the territory of the Czech Republic. According to the FAOSTAT (2017), the biggest barley producers in Europe are Spain, France, and Germany. For malting, varieties with a two-row ear type are prevailingly used in Europe; nevertheless, there are also six-row malting varieties. However, for malting, only the highest quality part of the production is used. The quality of malting barley is affected by a number of factors, the most important of which include a genotype and weather conditions during growing (Przulj et al., 2014). Farmers can therefore influence the quality of their production mainly by choosing a variety, to a lesser extent by agricultural engineering employed.
Malt contains various compounds that come directly from barley kernels or are formed during malting. The barley used in the malting process thus has a significant impact on the quality of the final product - beer. High malting quality of barley grain has not been unambiguously defined yet. Producers of malt and subsequently beer consider a quality raw material (barley grain, malt) the material that suits the technology of malt production or beer employed by them. The quality of malt can be characterized by dozens of different traits (Leistrumaitë and Paplauskienë, 2005).

Large grains with a uniform size above $2.5 \mathrm{~mm}$ are required to ensure uniform malting because large grains have a higher starch content and lower protein levels 
than smaller grains (Fox et al., 2006). One of the most important traits is the nitrogen content of a barley grain. The content of nitrogenous substances in a barley grain is easily influenced by the growing conditions and the agricultural engineering used, including the application of nitrogenous fertilizers (Condón et al., 2009). Higher contents of nitrogenous substances in a grain were found in years with dry and warm weather at the time of the grain maturation (Cativelli et al., 2011; Morgan and Riggs, 1981; Savin and Nicolas, 1996; Savin and Nicolas, 1999). High protein contents are in a strong correlation with a low starch content and thus low extract content (Fox et al., 2003). A key indicator of the malting quality is a malt extract, which is affected by a number of independent components (Przulj et al., 2013).

So far, breeding programs have used mainly high productivity varieties that come from a narrow gene pool. Further improvements in malting quality must rely on new combinations of genes and germplasm (Matthies et al., 2014). Plant genetic resources are an indispensable source of genetic variability and their use in breeding programs increases the chances for success in designing new productive cultivars with good quality properties (Bockelman et al., 2010), or even varieties more resistant to biotic and abiotic stressors.

The genetic diversity of barley is preserved both in gene banks (ex-situ) and in nature (in-situ). In the Czech Republic, the genetic resources are preserved within the National Program on Conservation and Utilization of Plant Genetic Resources and Agro-biodiversity. At present, a total of 3046 items are kept in the Collection of Spring Barley Genetic Resources and every year the collection is enriched with other newly bred varieties and genotypes that might be of an interest for breeders and researchers.

Figure 1 Average temperatures and sum of precipitation during the growing seasons 2010 and 2011

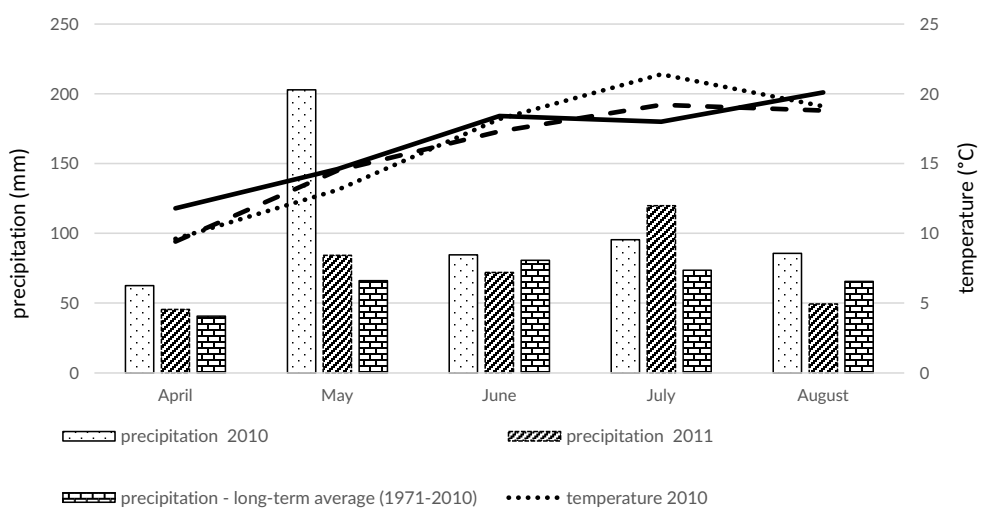

- - temperature - long-term average (1971-2010) —
The aim of this study was to evaluate the genetic resources selected from the collection of spring barley in terms of their malting characteristics and assess their potential for the use in breeding of malting varieties.

\section{Material and Methods}

The grain samples were obtained from the collection of the genetic resources of spring barley kept within the National Program on Conservation and Utilization of Plant Genetic Resources and Agro-biodiversity (MZe, 2017). An experimental set of 92 genetic resources of spring barley was grown on plots of $2.5 \mathrm{~m}^{2}$ after a previous crop of winter rape on the lands of the Agricultural Research Institute Kroměříž, Ltd. (49 $17^{\circ} \mathrm{N}, 17^{\circ} 22^{\prime} \mathrm{E}, 235$ m a.s.l.), average annual temperature $8.7^{\circ} \mathrm{C}$, average annual rainfall $559 \mathrm{~mm}$ in 2010-2011. The course of the weather during the growing seasons (2010 and 2011) is shown in Figure 1. No fungicides or morphoregulators were applied during the growing season. The set included hulled genotypes, both with a two-row and six-row type of the ear.

The graded grain samples $(2.5 \mathrm{~mm})$ weighing $500 \mathrm{~g}$ from the 2010 and 2011 harvest years were used for malting. Malts were prepared in a laboratory malting device (KWM Uničov, the Czech Republic) according to the methodology of Mitteleuropäische Brautechnische Analysenkommission (MEBAK, 2011) in the following conditions: $72 \mathrm{~h}$ steeping at $14{ }^{\circ} \mathrm{C}$ with $\mathrm{CO}_{2}$ exhaustion, steeping periods on $1^{\text {st }}$ day $5 \mathrm{~h}$, on $2^{\text {nd }}$ day $4 \mathrm{~h}$ and on $3^{\text {rd }}$ day $3 \mathrm{~h}$. Germination time was $72 \mathrm{~h}$ at $14{ }^{\circ} \mathrm{C}$. Pre-drying $12 \mathrm{~h}$ at $55^{\circ} \mathrm{C}$, kilning $4 \mathrm{~h}$ at $80^{\circ} \mathrm{C}$. In this study, the following parameters were monitored: starch content in barley grain (\%), barley bulk density $\left(\mathrm{g} / \mathrm{dm}^{3}\right)$, malt extract (\%), relative extract at $45^{\circ} \mathrm{C}(\%)$, Kolbach index (\%), diastatic power $(\mathrm{WK})$, apparent final attenuation (\%), friability (\%), $\beta$-glucans in wort $\left(\mathrm{mg} / \mathrm{dm}^{3}\right)$, wort haze measured at $12^{\circ}$ and $90^{\circ}$.The studied malting parameters (Table 2 ) were determined according to the methodologies of the EBC Analysis Committee (2010) and MEBAK (2011).

To process the results, the evaluated genetic resources were divided according to the areas of their breeding into five groups (Figure 2): A - Western Europe (Great Britain, Germany, the Netherlands, Denmark, France, Portugal); B - Central and Eastern Europe (former Czechoslovakia, the 
Czech Republic, Slovakia, Austria, Russia); C - North America (Canada, USA); D - South America and Australia (Bolivia, Argentina, Uruguay, Australia); E - Middle East and Africa (Israel, Egypt, Turkey). The studied genetic resources of individual groups are listed in Table 1, Table 2 and Table 3.

Statistica 12.0 was used for the statistical evaluation of the results.

\section{Results and Discussion}

The malting quality of barley varieties is evaluated on the basis of the level of degradation of cell walls, nitrogenous substances and starch. Further, some of sensory wort characters are also assessed. The average values of the important malting parameters recorded in the evaluated set of the genetic resources are given in Table 4 . The biggest variability was found in malt haze $\left(90^{\circ}\right)$, however, the six-row and two-row genotypes did not differ statistically significantly in this parameter (Table 5). The least variable parameter according to the coefficient of variation (CV) was apparent final attenuation $(\mathrm{CV}=2.70 \%)$.

\section{Level of cytolytic modification}

Cytolytic modification of malt is the result of the action of a whole complex of enzymes which break down hemicellulose inside the endosperm to simple sugars. The content of $\beta$-glucans in wort affects lautering and filtration time. In brewing, these $\beta$-glucans affect the amount of the obtained extract, and thus the brewing yield as well.

Malt friability is an important indicator of malt quality; it indirectly informs about the level of the cell wall degradation during malting. The values in the studied set ranged from $32.0 \%$ to $99.0 \%$, with an average value of 74.15\%. Prokeš et al. (2007) found a larger range of values in the set of $113 \mathrm{Czech}$ and foreign genetic sources (15.4\%-90.8\%), but the varieties with the lowest average value of friability were mainly the materials from Japan with a six-row ear and hulless caryopsis. In the studied set, the lowest value of malt friability (35.5\%) was found in Cebada Capa from Argentina (Table 3). In all groups with non-European genetic resources, the level of friability was lower than 75\% (Table 7). But even within these groups, genetic resources can be found close to the European varieties in this trait. For example, in the group of varieties from North America (C), malt friability of the varieties Conrad (80.5\%) and Long Glumes (78.0\%) approached the average values of the A and B groups of European varieties (78.7\% and 76.8\%). In the group of varieties from South America and Australia (D), Psaknon reached the average value of $76.0 \%$. The highest value of malt friability (94.5\%) was found in the German variety Germina (Table 1). In the studied set, both the individual years (Table 6) and the average values of two-row and six-row genotypes differed statistically significantly (Table 5). Strong positive correlations were found between friability and the Kolbach index $(r=0.84)$. An equally important correlation between friability and the malt extract $(r=0.81)$ was also found in a study of historical data of barley collections of an European origin performed by Matthies et al. (2014). Further, a strong negative correlation was recorded between friability and the content of $\beta$-glucans in wort $(r=-0.77)$. Šmić et al. (2015) found in their study of winter and spring barley an even higher statistically significant negative correlation between the content of $\beta$-glucans in wort and friability $(r=-0.93)$. The individual areas were divided into three statistically significant groups based on their friability values (Table 7). The lowest average values were found in groups $\mathrm{D}$ and $\mathrm{E}$ (49.8\% and 50.3\%), middle in group C (62.5\%), the highest then in groups A and B (78.69\% and $76.82 \%)$.

The $\boldsymbol{\beta}$-glucan content in wort is another very important parameter monitored within the malt evaluation as $\beta$-glucans increase the viscosity of sweet wort and inhibit filtration. The content of $\beta$-glucans in wort in the evaluated set of genotypes was highly variable ( $C V=60.59 \%$ ). As reported by Zheng et al. (2011), levels of $\beta$-glucans are influenced by both genetic and environmental factors; the genetic factors appear to be of greater importance (Zheng et al., 2011). Psota (2008) found only half the maximum content (60.0-628.0 mg/dm ${ }^{3}$ ) in his study compared to our data. Statistically significant differences were detected in this parameter both between the growing years 


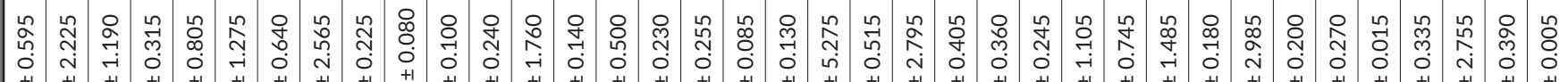

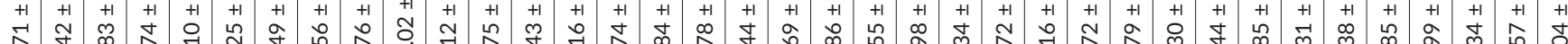

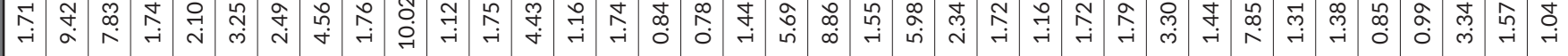

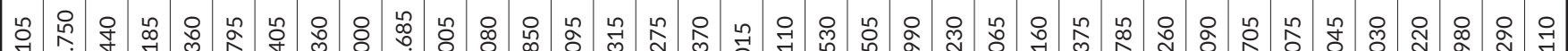

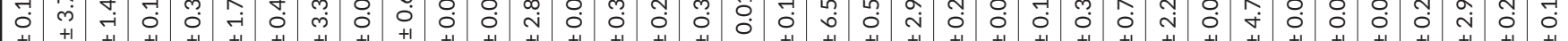
ס

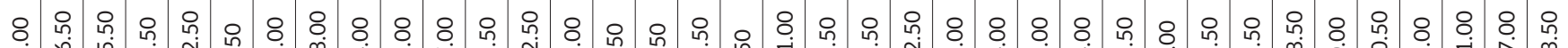

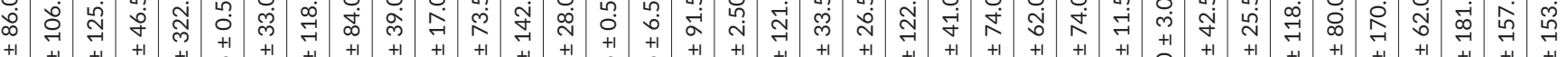

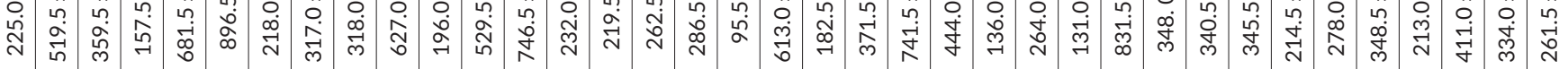

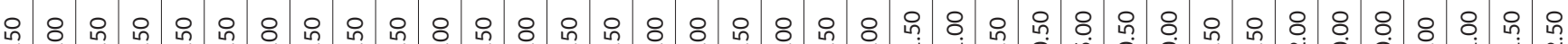

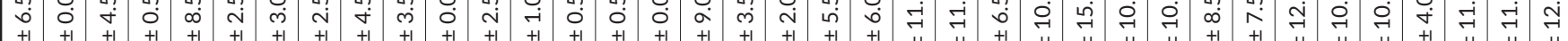

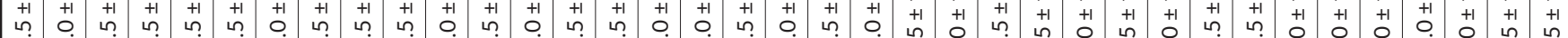

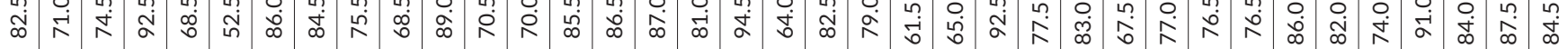

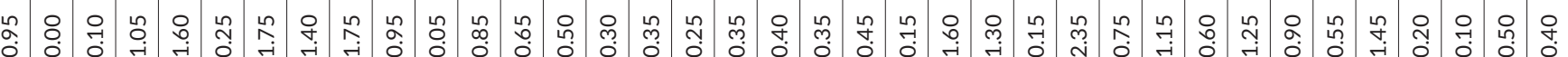

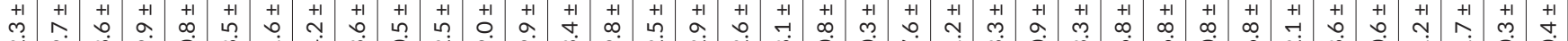

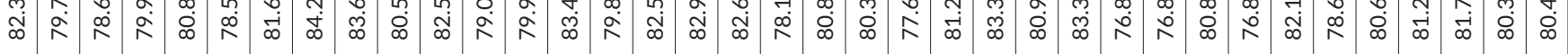

要,

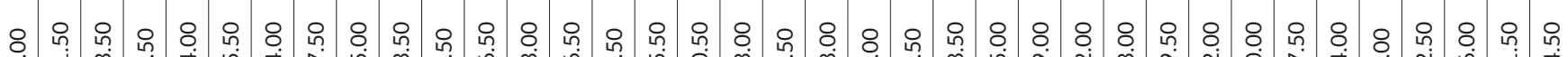

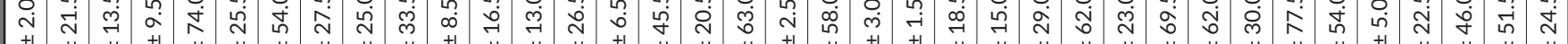

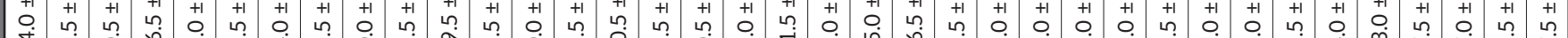

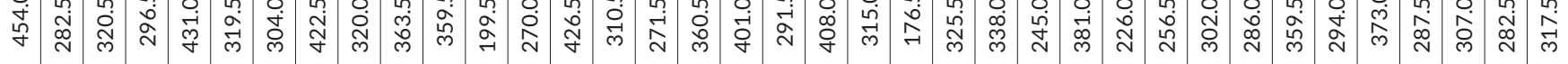

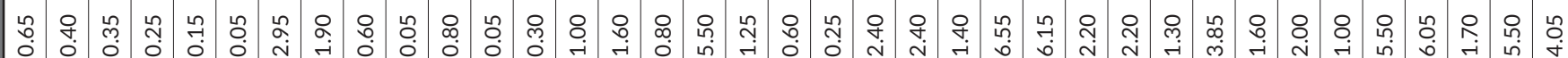

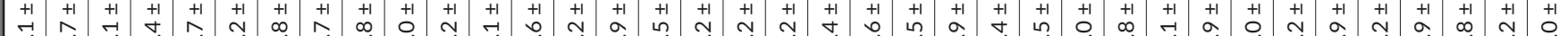
多

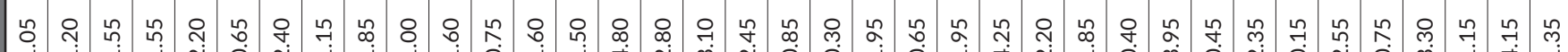

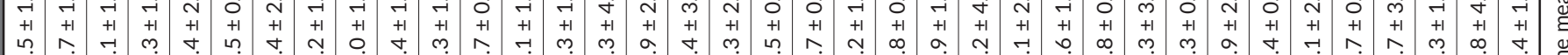

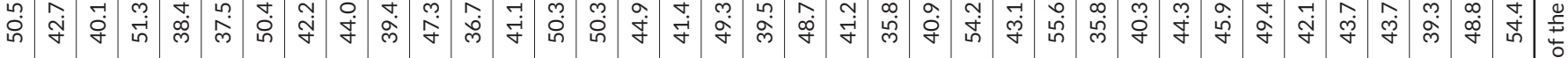

น

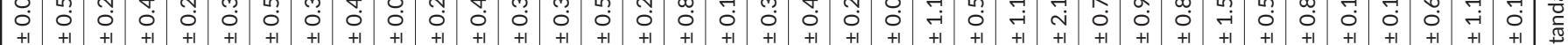

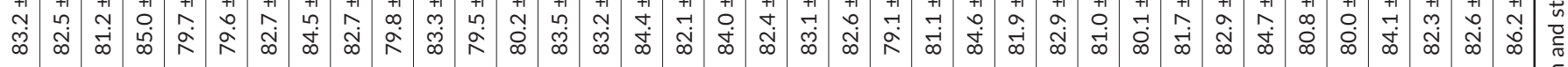

离 8 늠

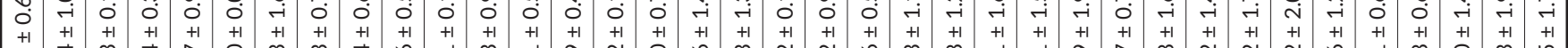

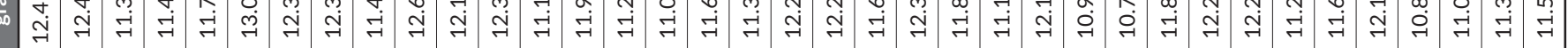

m 


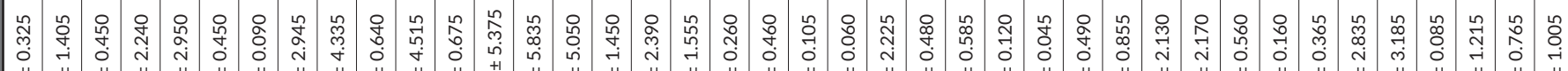

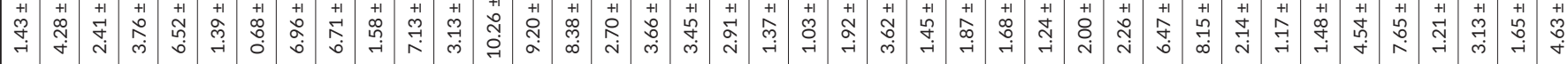

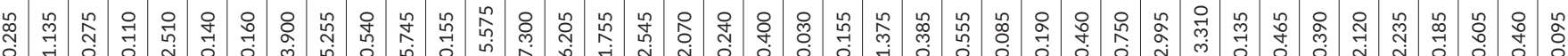

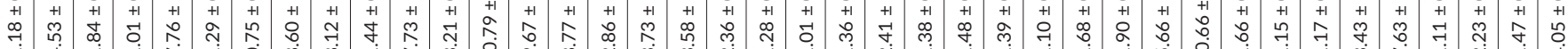

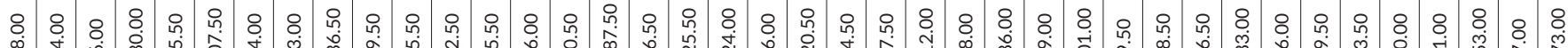

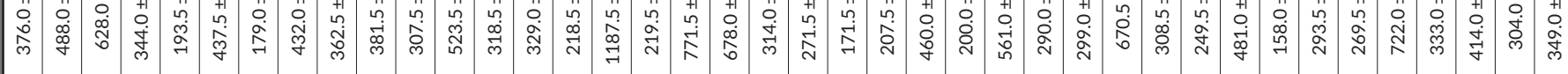

竞

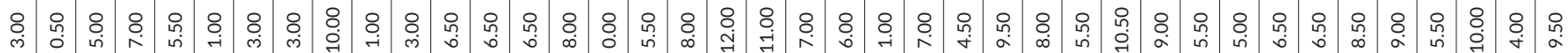

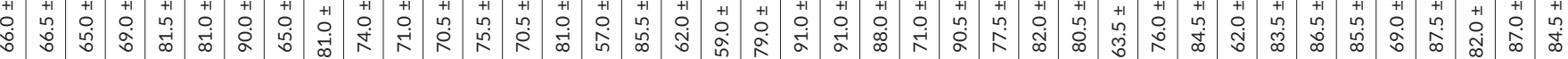

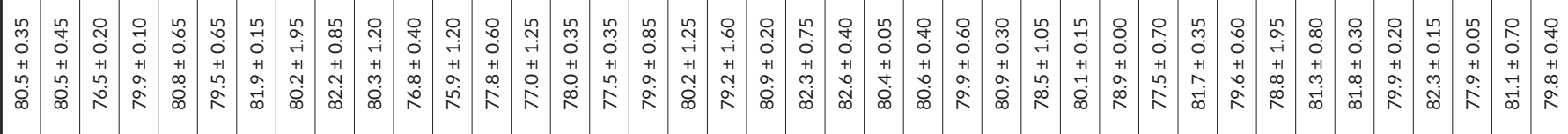

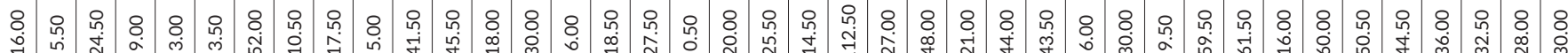

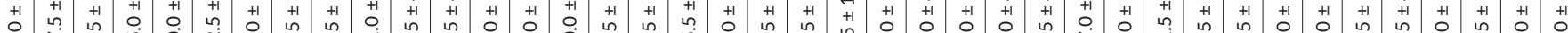

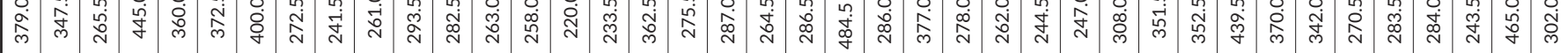

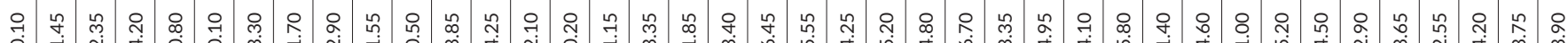

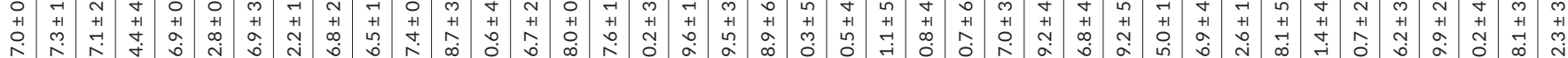

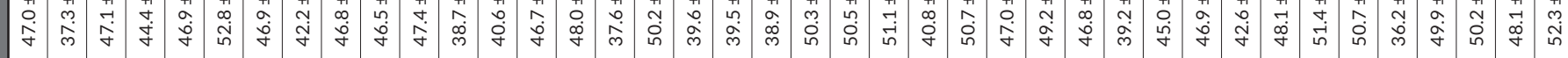

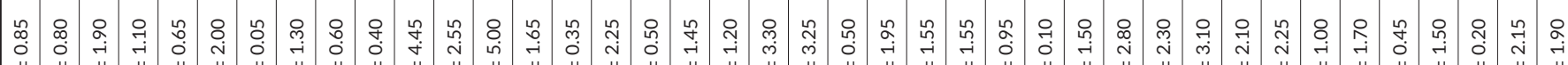

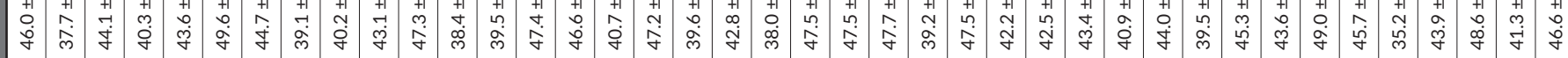

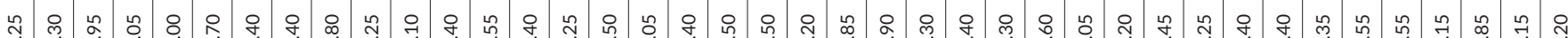

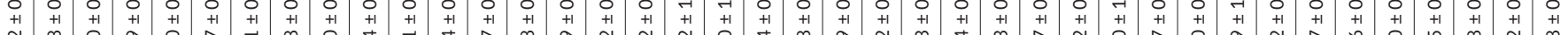

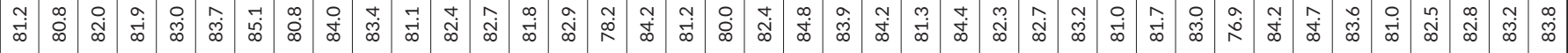

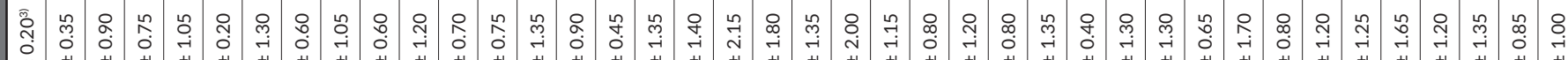

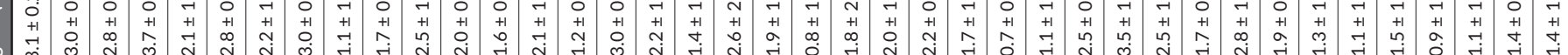

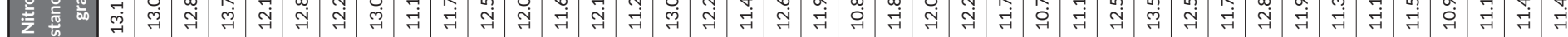




\begin{tabular}{|c|c|c|c|c|c|c|c|c|c|c|c|c|c|c|c|c|}
\hline $\mathbb{N}$ & 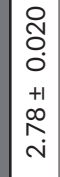 & 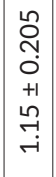 & $\begin{array}{c}\tilde{L} \\
\hat{N} \\
0 \\
+1 \\
+1 \\
0 \\
\infty \\
\dot{n}\end{array}$ & 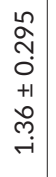 & 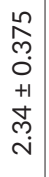 & $\left|\begin{array}{c}n \\
\alpha \\
n \\
0 \\
+1 \\
\hat{\alpha} \\
\infty \\
+i\end{array}\right|$ & $\begin{array}{c}n \\
0 \\
\tilde{m} \\
0 \\
+1 \\
\tilde{O} \\
\stackrel{N}{1}\end{array}$ & $\begin{array}{l}\stackrel{n}{m} \\
- \\
- \\
+1 \\
-1 \\
-1 \\
\rightarrow \\
\rightarrow\end{array}$ & 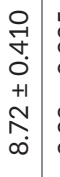 & 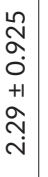 & $\begin{array}{c}\stackrel{8}{0} \\
\infty \\
i \\
+1 \\
\hat{\alpha} \\
\infty \\
\infty\end{array}$ & 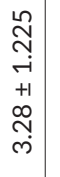 & $\begin{array}{l}n \\
0 \\
0 \\
0 \\
+1 \\
+1 \\
0 \\
n \\
\rightarrow \\
+i\end{array}$ & $\begin{array}{l}0 \\
\stackrel{0}{ } \\
0 \\
0 \\
+1 \\
+1 \\
\\
\end{array}$ & 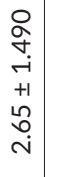 & \\
\hline & 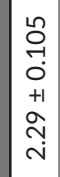 & $\begin{array}{l}n \\
\delta \\
\dot{0} \\
+1 \\
\tilde{O} \\
0 \\
0\end{array}$ & 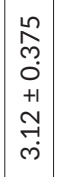 & 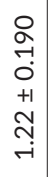 & $\begin{array}{l}0 \\
0 \\
0 \\
0 \\
+1 \\
+ \\
\vec{i} \\
\vec{i}\end{array}$ & $\mid \begin{array}{c}n \\
\infty \\
0 \\
0 \\
+1 \\
+1 \\
\stackrel{N}{\rightarrow}\end{array}$ & $\begin{array}{l}n \\
\alpha \\
0 \\
0 \\
+1 \\
0 \\
0 \\
\stackrel{+}{r}\end{array}$ & 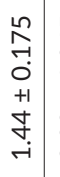 & $\begin{array}{l}n \\
0 \\
0 \\
+ \\
+ \\
+ \\
8 \\
\infty\end{array}$ & $\begin{array}{l}0 \\
\stackrel{2}{a} \\
\dot{0} \\
+1 \\
\text { +1 } \\
\dot{v}\end{array}$ & 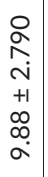 & 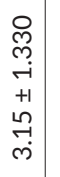 & $\begin{array}{c}\stackrel{g}{d} \\
\dot{d} \\
+ \\
+1 \\
\dot{f} \\
\dot{r}\end{array}$ & 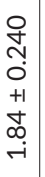 & 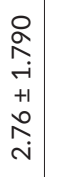 & \\
\hline & $\begin{array}{l}8 \\
0 \\
+1 \\
+1 \\
0 \\
\dot{a} \\
r\end{array}$ & 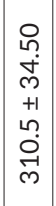 & $\begin{array}{l}8 \\
0 \\
0 \\
0 \\
+1 \\
0 \\
i \\
\hat{N}\end{array}$ & 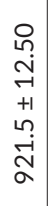 & 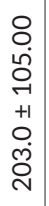 & $\begin{array}{c}8 \\
0 \\
0 \\
0 \\
+1 \\
0 \\
\dot{\rho} \\
\sim\end{array}$ & 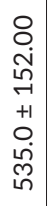 & 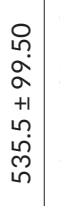 & 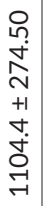 & 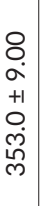 & 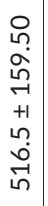 & $\begin{array}{l}0 \\
n \\
+ \\
a \\
+1 \\
n \\
0 \\
0 \\
0 \\
0\end{array}$ & 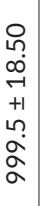 & 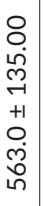 & 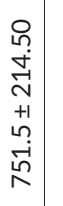 & \\
\hline & 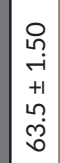 & $\mid \begin{array}{c}0 \\
i n \\
n \\
+1 \\
1 \\
0 \\
0 \\
0\end{array}$ & 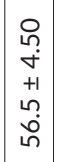 & $\begin{array}{l}0 \\
\stackrel{n}{0} \\
\rightarrow \\
+1 \\
n \\
0 \\
0 \\
\infty\end{array}$ & 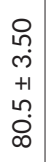 & $\mid \begin{array}{c}8 \\
0 \\
0 \\
+1 \\
+1 \\
0 \\
\infty \\
1\end{array}$ & $\begin{array}{l}8 \\
0 \\
+1 \\
0 \\
\text { ஸे }\end{array}$ & $\begin{array}{c}8 \\
\dot{m} \\
+1 \\
0 \\
\dot{\gamma} \\
\dot{\gamma}\end{array}$ & 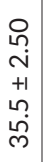 & $\begin{array}{l}8 \\
8 \\
\dot{1} \\
+1 \\
0 \\
\dot{0} \\
\end{array}$ & $\begin{array}{l}0 \\
i n \\
\alpha \\
+1 \\
n \\
\tilde{f} \\
f\end{array}$ & $\begin{array}{l}8 \\
\dot{0} \\
+1 \\
0 \\
i \\
i\end{array}$ & 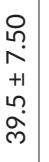 & 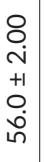 & 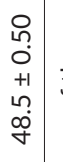 & \\
\hline 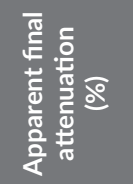 & 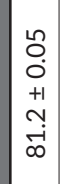 & $\mid \begin{array}{c}\llcorner \\
\infty \\
0 \\
0 \\
+1 \\
-1 \\
\dot{-1} \\
\infty\end{array}$ & 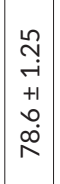 & $\begin{array}{l}n \\
\alpha \\
0 \\
+1 \\
o \\
o \\
\propto 0 \\
\wedge\end{array}$ & 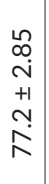 & $\mid \begin{array}{c}8 \\
0 \\
0 \\
+1 \\
n \\
\dot{0} \\
\infty\end{array}$ & $\begin{array}{l}0 \\
9 \\
0 \\
+1 \\
0 \\
0 \\
\triangleright \\
\triangleright\end{array}$ & $\left|\begin{array}{c}0 \\
m \\
i \\
+1 \\
\infty \\
0 \\
i \\
i\end{array}\right|$ & $\begin{array}{l}0 \\
0 \\
0 \\
+1 \\
-1 \\
-1 \\
\end{array}$ & 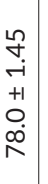 & 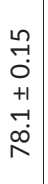 & 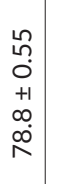 & 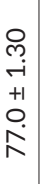 & $\begin{array}{l}8 \\
0 \\
0 \\
+1 \\
\stackrel{1}{1} \\
\end{array}$ & 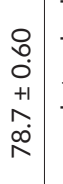 & \\
\hline & $\begin{array}{l}8 \\
\dot{T} \\
+ \\
+1 \\
0 \\
\infty \\
0 \\
m\end{array}$ & 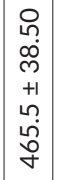 & $\begin{array}{c}8 \\
0 \\
0 \\
+1 \\
0 \\
\omega \\
0 \\
0\end{array}$ & 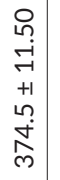 & 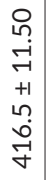 & $\left|\begin{array}{c}0 \\
1 \\
m \\
\rightarrow \\
+1 \\
n \\
n \\
-1 \\
m\end{array}\right|$ & 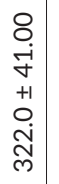 & $\begin{array}{c}8 \\
\dot{b} \\
\dot{q} \\
+1 \\
0 \\
\tilde{~} \\
\delta \\
\dot{e}\end{array}$ & $\begin{array}{l}8 \\
\stackrel{1}{+} \\
+ \\
+1 \\
\circ \\
\dot{+} \\
\stackrel{2}{*}\end{array}$ & 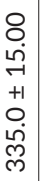 & 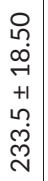 & $\begin{array}{c}\stackrel{8}{0} \\
\dot{\sim} \\
+1 \\
+1 \\
\infty \\
\infty \\
\infty \\
\sim\end{array}$ & $\begin{array}{l}0 \\
0 \\
0 \\
+1 \\
+ \\
\infty \\
\stackrel{0}{0} \\
\stackrel{n}{n}\end{array}$ & 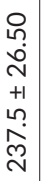 & 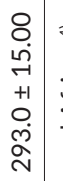 & \\
\hline & $\begin{array}{l}\stackrel{n}{2} \\
\sim \\
+ \\
+1 \\
o \infty \\
\dot{+}\end{array}$ & $\begin{array}{c}0 \\
0 \\
0 \\
+1 \\
+1 \\
\tilde{g} \\
\dot{q} \\
\end{array}$ & $\begin{array}{l}\stackrel{0}{N} \\
\text { d } \\
+1 \\
0 \\
\dot{q} \\
\dot{q}\end{array}$ & $\mid \begin{array}{c}\stackrel{o}{d} \\
\stackrel{1}{+} \\
+1 \\
\stackrel{m}{m}\end{array}$ & 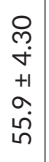 & 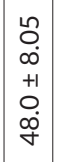 & 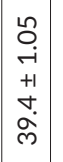 & 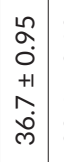 & 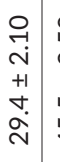 & 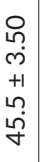 & $\begin{array}{l}\stackrel{8}{0} \\
\dot{+} \\
+1 \\
\tilde{N} \\
\tilde{m}\end{array}$ & $\begin{array}{l}n \\
\infty \\
+ \\
+1 \\
+1 \\
\infty \\
\infty \\
\infty\end{array}$ & 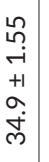 & $\begin{array}{l}\stackrel{o}{o} \\
\dot{+} \\
+1 \\
+ \\
\dot{q} \\
\dot{q}\end{array}$ & $\begin{array}{l}\tilde{n} \\
\tilde{m} \\
0 \\
+1 \\
+ \\
\tilde{j} \\
\tilde{m}\end{array}$ & \\
\hline$\approx \sigma_{\pi}$ & $\begin{array}{l}8 \\
0 \\
0 \\
+1 \\
0 \\
0 \\
\dot{\sigma}\end{array}$ & $\begin{array}{c}\stackrel{8}{ } \\
i \\
+1 \\
\dot{j} \\
\dot{q}\end{array}$ & $\begin{array}{c}0 \\
\infty \\
i \\
++ \\
i n \\
\dot{p} \\
\dot{\sigma}\end{array}$ & $\begin{array}{c}0 \\
\stackrel{0}{0} \\
0 \\
+1 \\
0 \\
\hat{m}\end{array}$ & 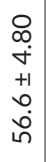 & $\begin{array}{c}0 \\
\tilde{m} \\
\dot{p} \\
+1 \\
\dot{w} \\
\dot{q} \\
\dot{q}\end{array}$ & $\left|\begin{array}{c}0 \\
o \\
\dot{2} \\
+1 \\
\stackrel{\vec{v}}{+}\end{array}\right|$ & $\begin{array}{c}0 \\
0 \\
0 \\
+1 \\
+1 \\
\infty \\
m \\
0\end{array}$ & 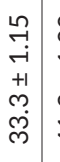 & 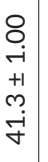 & $\begin{array}{l}\stackrel{?}{f} \\
0 \\
0 \\
+1 \\
\dot{1} \\
\dot{m}\end{array}$ & $\begin{array}{l}8 \\
\dot{i} \\
+1 \\
+ \\
\dot{\sigma} \\
\dot{m}\end{array}$ & $\begin{array}{c}\stackrel{n}{\sim} \\
\underset{+}{+} \\
+ \\
+ \\
\dot{q}\end{array}$ & 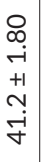 & 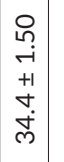 & \\
\hline & 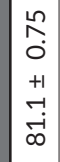 & 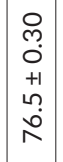 & $\begin{array}{c}0 \\
0 \\
0 \\
0 \\
+1 \\
0 \\
0 \\
\infty\end{array}$ & $\mid \begin{array}{c}\stackrel{0}{1} \\
\stackrel{i}{1} \\
+1 \\
\stackrel{i}{i} \\
\end{array}$ & $\begin{array}{c}n \\
? \\
0 \\
+ \\
+1 \\
\dot{1} \\
\dot{N}\end{array}$ & $\begin{array}{c}\tilde{n} \\
\tilde{D} \\
+1 \\
+1 \\
\sim \\
\infty \\
\infty\end{array}$ & 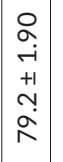 & 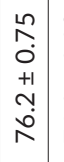 & 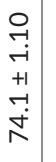 & $\begin{array}{c}\stackrel{n}{0} \\
0 \\
+1 \\
+1 \\
\dot{j} \\
\infty\end{array}$ & $\begin{array}{l}0 \\
\stackrel{0}{0} \\
0 \\
+1 \\
+ \\
\dot{R} \\
\stackrel{n}{n}\end{array}$ & 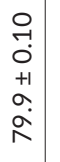 & 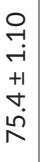 & 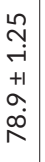 & $\begin{array}{c}0 \\
0 \\
0 \\
+1 \\
0 \\
0 \\
1 \\
1\end{array}$ & $c$ \\
\hline 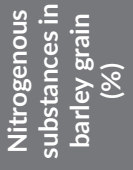 & 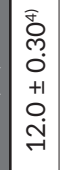 & 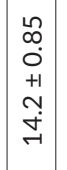 & 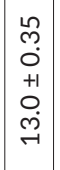 & 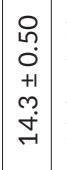 & 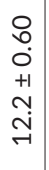 & $\begin{array}{l}0 \\
m \\
i \\
+1 \\
\stackrel{1}{i} \\
\vec{i}\end{array}$ & 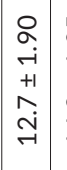 & 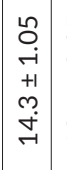 & $\begin{array}{l}\stackrel{n}{m} \\
\dot{0} \\
+ \\
+1 \\
\dot{0} \\
\stackrel{m}{-}\end{array}$ & 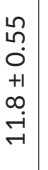 & 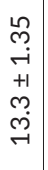 & 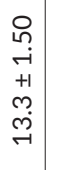 & $\begin{array}{c}0 \\
0 \\
0 \\
+1 \\
+1 \\
0 \\
\dot{0} \\
\end{array}$ & 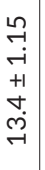 & $\begin{array}{l}\stackrel{P}{1} \\
\stackrel{i}{+} \\
+1 \\
\stackrel{9}{9} \\
\rightarrow\end{array}$ & to \\
\hline & $u$ & $u$ & $u$ & $u$ & $u$ & $u$ & $u$ & 0 & o & O & ० & ш & ш & ш & س & \\
\hline & 0 & ○ & 0 & 0 & $N$ & $N$ & $N$ & $N$ & 0 & $N$ & 0 & $N$ & $N$ & $N$ & $N$ & \\
\hline 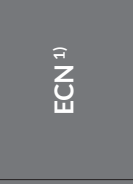 & 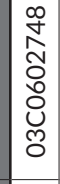 & $\begin{array}{l}0 \\
\vdots \\
0 \\
o \\
o \\
0 \\
0 \\
0\end{array}$ & $\mid \begin{array}{l}0 \\
0 \\
\vdots \\
0 \\
o \\
0 \\
0 \\
0\end{array}$ & $\mid \begin{array}{c}0 \\
o \\
\tilde{z} \\
0 \\
0 \\
\mathcal{ల} \\
0\end{array}$ & 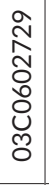 & $\mid \begin{array}{l}a \\
\hat{w} \\
0 \\
0 \\
0 \\
0 \\
0\end{array}$ & 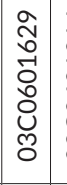 & $\mid \begin{array}{l}\vec{J} \\
0 \\
0 \\
0 \\
0 \\
0 \\
o\end{array}$ & $\begin{array}{l}\circ \\
2 \\
0 \\
0 \\
0 \\
0 \\
0\end{array}$ & $\begin{array}{l}0 \\
\text { o. } \\
\text { - } \\
0 \\
0 \\
0 \\
0\end{array}$ & $\begin{array}{l}-1 \\
0 \\
0 \\
0 \\
0 \\
0 \\
0 \\
0\end{array}$ & 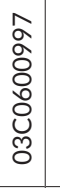 & 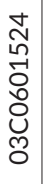 & $\begin{array}{l}\hat{N} \\
0 \\
0 \\
0 \\
0 \\
0 \\
0\end{array}$ & $\begin{array}{l}a \\
o \\
o \\
\tilde{o} \\
0 \\
0 \\
\tilde{o}\end{array}$ & | \\
\hline 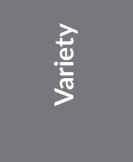 & 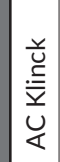 & $\mathscr{\infty}$ & $\mid \begin{array}{l}\stackrel{\check{L}}{0} \\
\stackrel{\bar{g}}{0}\end{array}$ & $\mid \begin{array}{l}\stackrel{\infty}{0} \\
\stackrel{1}{U} \\
\bar{U}\end{array}$ & & $\left|\begin{array}{l}\frac{y}{0} \\
0 \\
00 \\
\overline{0} \\
\end{array}\right|$ & $\bar{\Sigma} \mid$ & 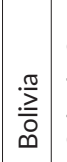 & $\begin{array}{l}\frac{\pi}{0} \\
0 \\
0 \\
\frac{\pi}{0} \\
\frac{0}{0} \\
0\end{array}$ & & $\simeq$ & 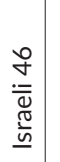 & 气 & & 产 & \\
\hline
\end{tabular}

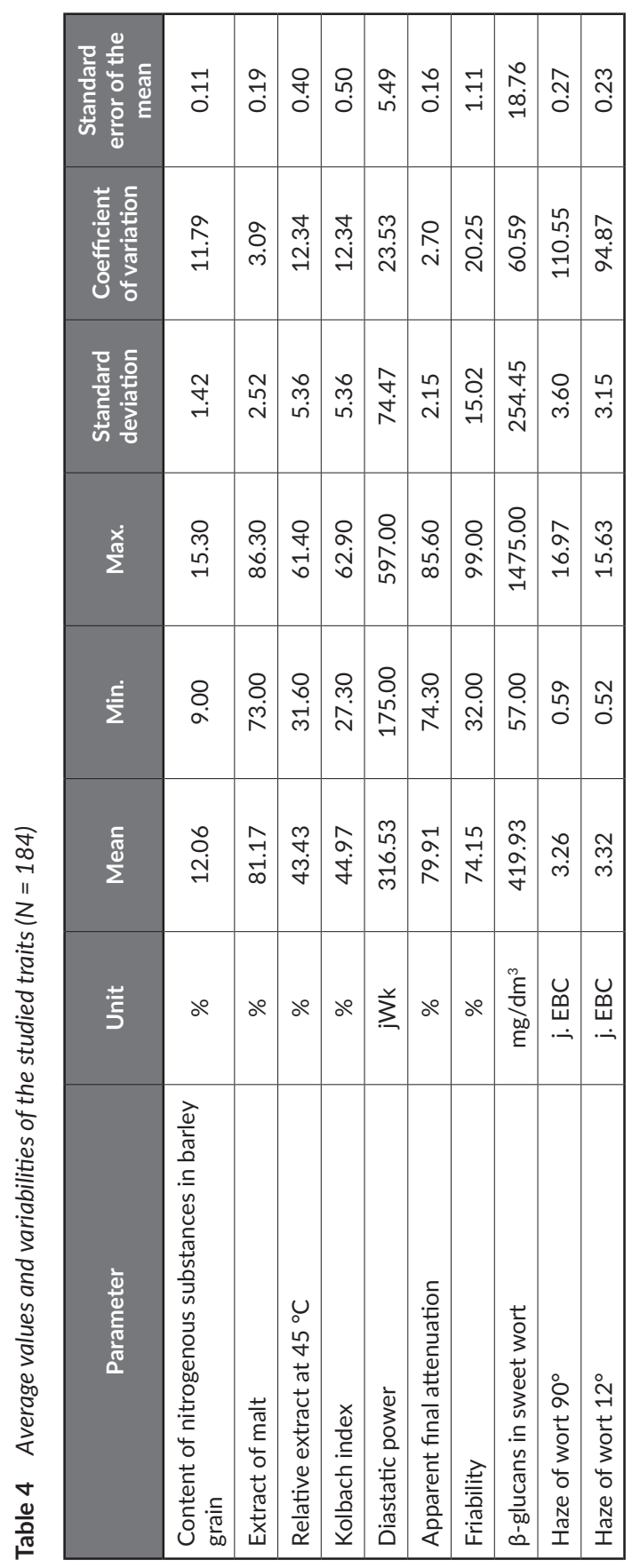




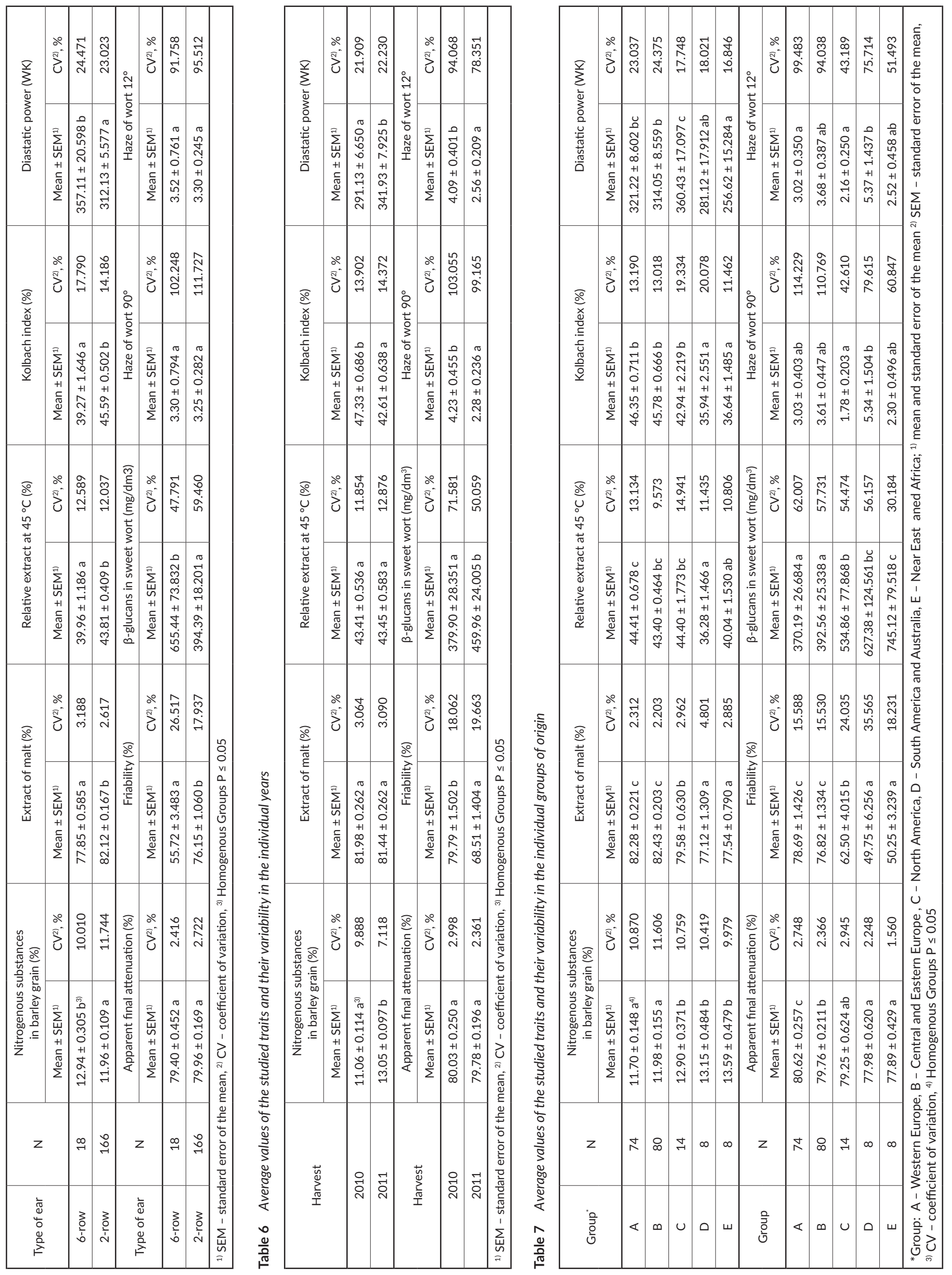


(379.90 mg/dm ${ }^{3}$ and $459.96 \mathrm{mg} / \mathrm{dm}^{3}$ in 2010 and 2011, resp.) and genetic resources with the two-row and sixrow type of the ear $\left(394.39 \mathrm{mg} / \mathrm{dm}^{3}\right.$ and $655.44 \mathrm{mg} / \mathrm{dm}^{3}$, resp.). The average value of the set $\left(419.93 \mathrm{mg} / \mathrm{dm}^{3}\right)$ was increased mainly by the genotypes of the non-European origin groups (C, D, E); this was caused by a higher representation of the six-row varieties in these groups. But even within these groups, genetic resources with low levels of $\beta$-glucans in sweet wort could be found: for example, Conrad (203.0 mg/dm³ ), Long Glumes (239.0 mg/ $\mathrm{dm}^{3}$ ) and Bolron (310.5 mg/dm ${ }^{3}$ ) in the group of varieties from North America (C), Psaknon $\left(353.0 \mathrm{mg} / \mathrm{dm}^{3}\right)$ in the group of varieties from South America and Australia (D) Table 3. The highest average value $\left(745.12 \mathrm{mg} / \mathrm{dm}^{3}\right)$ was found in group E (Middle East and Africa). However, the variety with the highest $\beta$-glucan content belonged to the group of varieties from Central and Eastern Europe: the Czech breeding line KM 1192 (1187.5 mg/dm³). The lowest average value of $\beta$-glucan content in sweet wort was measured in the German variety Germina $\left(95.5 \mathrm{mg} / \mathrm{dm}^{3}\right)$. A high content of $\beta$-glucans in wort had a statistically significant negative effect on the malt extract, the relative extract at $45^{\circ} \mathrm{C}$, the Kolbach index, the apparent final attenuation and friability (Table 8). For this reason, breeders try to breed varieties with the lowest possible $\beta$-glucan content in the grain.

\section{Level of proteolytic modification}

Proteolytic modification of malt is a process of degradation of proteins by hydrolytic enzymes. The content of nitrogenous substances in barley caryopses and the degree of their modification during malting affect the beer foam capacity, wholesomeness of beer, occurrence of chill haze, filterability and colour of wort.
The content of nitrogenous substances in barley grain is one of the most important characteristics. In the studied set of genotypes, the values ranged from $9.0 \%$ to $15.3 \%$, with the average of $12.1 \%$ (Table 4 ). Statistically significant differences between the two-row and six-row varieties (Table 5) and the growing years (Table 6) were recorded. All other groups (C, D and E) differed statistically significantly from the European varieties (groups A and B) in this parameter, but they did not differ from each other (Table 7). In the group of varieties from South America and Australia (D), Psaknon (11.8\%) differed from the group with its average of nitrogen content in barley grain (13.2\%); in this parameter it was comparable with the European varieties (groups A and B - the average values of $11.7 \%$ and $12.0 \%$, resp.). Within the studied set, statistically significant positive correlations were found between the content of nitrogenous substances in the grain and diastatic power $(r=0.36)$ and $\beta$-glucan content in wort $(r=0.37)$. Further, negative correlations were found in connection with the malt extract $(r=-0.53)$, Kolbach index $(r=-0.60)$, apparent final attenuation $(r=-0.27)$, friability $(r=-0.73)$ (Table 8).

The Kolbach index represents the degree of protein modification. Statistically significant differences were found both between the two-row and six-row genotypes and between the studied years (Table 5, Table 6). The values ranged from 27.3 to $62.9 \%$. Amabile et al. (2014) reported the range of values of the Kolbach index in a set of 30 malting two-row and six-row barley mainly from the area of South America in the range of 38.7-64.8\%. In the observed set, the group from South America and Australia (35.9\%) together with the group from the Middle East and Africa (36.6\%) differed statistically significantly in this parameter from the other groups (Table 7).

Table 8 Matrix of correlation between the studied traits in the set of spring barley genetic resources

\begin{tabular}{|c|c|c|c|c|c|c|c|c|c|c|}
\hline Parameter & NS & E & RE45 & KI & DP & AFA & $\mathbf{F}$ & BG & $\mathrm{H} 90$ & H12 \\
\hline NS & - & & & & & & & & & \\
\hline E & $\left.0.53^{* * *} 2\right)$ & - & & & & & & & & \\
\hline RE45 & -0.16 & $\left.0.52^{* * *} 2\right)$ & - & & & & & & & \\
\hline $\mathrm{KI}$ & $-0.60^{* * *}$ & $0.74^{* * *}$ & $0.77^{* * *}$ & - & & & & & & \\
\hline DP & $0.36^{* * *}$ & 0.09 & $0.29^{* * *}$ & 0.08 & - & & & & & \\
\hline AFA & $-0.27^{* * *}$ & $0.44^{* * *}$ & 0.24 & $0.36^{* * *}$ & $0.33^{* * *}$ & - & & & & \\
\hline $\mathrm{F}$ & $-0.73^{* * *}$ & $0.85^{* * *}$ & $0.54^{* * *}$ & $0.84^{* * *}$ & 0.03 & $0.46^{* * *}$ & - & & & \\
\hline BG & $0.37^{* * *}$ & $-0.63^{* * *}$ & $-0.58^{* * *}$ & $-0.72^{* * *}$ & -0.22 & $-0.43^{* * *}$ & $-0.77^{* * *}$ & - & & \\
\hline $\mathrm{H} 90$ & -0.18 & -0.08 & $-0.24^{* * *}$ & -0.09 & $-0.25^{* * *}$ & $-0.26^{* * *}$ & -0.02 & 0.09 & - & \\
\hline $\mathrm{H} 12$ & -0.15 & -0.11 & -0.22 & -0.1 & -0.23 & $-0.32^{* * *}$ & -0.05 & 0.1 & $0.97^{* * *}$ & - \\
\hline
\end{tabular}


According to Hartman et al. (2017), the optimal values range from 42 to $48 \%$. This range was also met by the average values found in the varieties belonging to groups A (46.4\%), B (45.8\%) and C (42.9\%) - Table 7. The variety Psaknon from South America and Australia (group D) approached with its average values (45.5\%) the averages of groups A and B. The lowest average values were measured for the Argentine variety Cebada Capa (29.4\%), the American breeding line CI 7584 (31.7\%) and Uruguayan Ricardo (32.2\%). The highest values were found in the West European varieties Biathlon (57.4\%), Mauritia (56.4\%), and Westminster (56.0\%) - Table 1. In the evaluated set of genotypes, a strong negative correlation was detected between the Kolbach index and the content of $\beta$-glucans in wort $(r=-0.72)$. A similar correlation was also found by Jin et al. (2011) and Amabile et al. (2014).

Relative extract at $45^{\circ} \mathrm{C}$ gives the activity of proteolytic enzymes and $\beta$-amylase. In the monitored group, the value of the relative extract ranged from $31.6 \%$ to $61.4 \%$. The lowest average values of this parameter were found in the South American varieties Ricardo and Cebada Capa (32.1\% and 33.3\%) and the Turkish variety Turk (34.4\%). The highest average values were reached by the Canadian variety Conrad (56.6\% - Table 3) and the malting varieties from Great Britain - Westminster and NFC Tipple (54.4\% and 55.6\%) - Table 1.

The values of the relative extract at $45{ }^{\circ} \mathrm{C}$ differed statistically significantly in the two-row and six-row varieties. Only the group of varieties from South America and Australia (36.3\%) differed statistically significantly from the genotypes from Western Europe (44.4\%) in this trait (Table 7). A strong positive correlation was found between the relative extract at $45^{\circ} \mathrm{C}$ and the Kolbach in$\operatorname{dex}(\mathrm{r}=0.77)$. Other significant positive correlations of the relative extract at $45{ }^{\circ} \mathrm{C}$ with other characteristics were found to be mild, with the values ranging from 0.24 to 0.53 (Table 8). Slight significant negative correlations were found between this parameter and the content of $\beta$-glucans in wort $(r=-0.58)$ and wort haze at $90^{\circ}$.

\section{Level of amylolytic modification}

Amylolytic modification informs about the activity of amylolytic enzymes that cleave starch. The intensity of amylolytic enzymes is reflected in the most important malt trait, the extractivity of malt. It is the percentage of malt that passes into solution under the conditions of mashing. The amount of fermentable sugars is essential for fermentation, for this reason, amylolytic modification can be characterized by the apparent final attenuation.

The value of diastatic power in the studied genetic resources ranged from 175.0 WK to 597.0 WK. Psota (2008) and Jin et al. (2011) reported smaller ranges of values for this parameter (226-442 WK and 254-516 WK, resp.). The probable reason is that, in this study, a more variable set of materials was studied. A statistically significant difference between the individual years was proven. In 2010 , genetic resources with a two-row type of the ear had a statistically significantly lower level of diastatic power compared to the six-row genotypes (Table 5). The group of genotypes "B" originating from Central Europe differed statistically significantly in this trait from those from North America (group C) and the Middle East and Africa (group E) - Table 7. Furthermore, in the values of diastatic power, the two-row genotypes differed statistically significantly from the six-row genotypes. Hartman et al. (2017) reported the optimal value of this parameter of 220-300 WK. Many European varieties did not meet this range, such as Antigone (454 WK), Brenda (423 WK), Class (427 WK), Germina (401 WK), Amulet (445 WK), Nitran ( $485 \mathrm{WK}$ ), and others (Tables 1 and 2). On the contrary, this range was met by a number of genetic resources from the groups of the non-European varieties - Cebada Capa (254 WK, group D), Ricardo (234 WK, group D), Israel 46 (288 WK, group E), Palestine 10 (238 WK, group E), Turk (293 WK, group E). The lowest average value of diastatic power was found in the British variety Magnum (177 WK); the highest in the already mentioned Slovak malting variety Nitran (485 WK) - Table 2.

The values of the extract content in the malt dry matter in the studied set ranged from 73.0 to $86.3 \%$ (Table 4). The boundary average values of this parameter for the individual varieties were $74.1 \%$ (the lowest), again for the Argentine variety Cebada Capa (Table 3), and $86.2 \%$ (the highest) for the British variety Westminster (Table 1). The individual monitored years did not differ in the content of malt extract (Table 6). The two-row genotypes had statistically significantly higher contents of malt extract $(82.1 \%)$ compared to the six-row ones (77.9\%). In terms of groups by origin, the genotypes were divided into three groups according to the content of malt extract, which differed statistically significantly (Table 7). The genotypes from groups D and E had the lowest $(77.1 \%$ and $77.5 \%)$, genetic resources from group C medium (79.6\%) and materials from groups A and B statistically significantly the highest (82.4\% and $82.3 \%)$ average values of the malt extract content. The acceptable range of malt extract values of 81.5 (283.0\%, as reported by Hartman et al. (2017), was found only in the genotypes originating from Europe (groups A and B), where breeding for malting quality has been conducted since the 19th century and quality sources have been available. However, in group D, the highest average content of extract in the malt dry matter was found in Psaknon $(82.9 \%)$, which by this value exceeded the average 
values of the groups of the European varieties - A and B $(82.3 \%$ and $82.4 \%)$. In the studied set, a positive highly significant correlation between the malt extract content and friability was found $(r=0.85)$. However, Mathies et al. (2014), who studied a group of 174 European spring and winter barley over 25 years, did not find such a correlation in their study.

Apparent final attenuation was the least variable parameter (Table 4). Its values ranged from $74.3 \%$ to $85.6 \%$. A similar range of values was also reported by Psota et al. (2017) for a set of 35 old and modern varieties of a Slovak origin (73.5-82.5\%). A larger range of values of this trait was found by Prokeš et al. (2007) in a set of 113 Czech and foreign varieties: from $58.6 \%$ to $84.1 \%$. However, these sets included both hulled and hulless genetic resources. Neither the individual years nor the two-row and six-row genotypes differed in this trait. A lower average value of this parameter than in Cebada Capa (76.1\%) was found only in Czech Granát (75.9\%). The highest average value was detected in the group of varieties from Western Europe - group A (80.6\%), which corresponds to the highest average values of this trait found within the individual varieties - Brenda $(84.2 \%$, Germany), Calgary (83.6\%, France) and Class (83.4\%, Great Britain) - Table 1. This group (A) differed statistically significantly from all other groups of the varieties, even from those of the origin from Central and Eastern Europe (group B). Hartman et al. (2017) reported the optimal range of this trait from $79.0-82.0 \%$. The average values of groups $\mathrm{D}$ and $\mathrm{E}$ were below the level of this minimum limit value. However, a variety that complied with this range was also detected in group D; it was the variety Bolivia (79.8\%). In this study, a positive correlation was found between the achievable degree of the apparent final attenuation and friability $(r=0.46)$, similarly as found by Matthies et al. (2014), $r=0.40$.

\section{Sweet wort sensory characters}

Of the sweet wort sensory characters, only the haze of sweet wort was assessed. Wort clarity is considered a sign of well modified barley. According to Prokeš et al. (2007) the haze of clear wort should reach the values up to $2.5 \mathrm{EBC}$. In the studied groups, the haze of sweet wort was measured at angles of $12^{\circ}$ and $90^{\circ}$. Both these traits showed the highest variability of all the studied parameters (Table 4). The genetic resources with the two-row and six-row ear type did not differ in their average values of haze (at $12^{\circ}$ and $90^{\circ}$ ), but statistically significant differences were found in the individual years (Table 6). Similar differences in both traits were found between the individual groups, where only groups $C$ and $D$, i.e. genetic resources originating from North America, differed from genetic resources from South America and Australia. The lowest values of wort haze at $12^{\circ}$ and $90^{\circ}$ were reached by the Czech variety Bojos (0.68 and 0.75 ), which was registered already in 2005 and is still greatly demanded (Psota et al. 2020). The highest value of wort haze at $12^{\circ}$ was found in the Czech variety Heris (10.3) and at $90^{\circ}$ in the German variety Aphrodite (11.3).

\section{Conclusion}

The breeding programs of malting varieties in Western and Central Europe reflect the requirements of the local beer producers. As genetically related varieties are frequently used, suitable genotypes coming from the world's collections of genetic resources might bring new genetic diversity to European breeding. The obtained results show that even the non-European groups of varieties can offer promising genetic resources that could enhance the genetic variability in breeding of European malting varieties; such as the Australian two-row variety Psaknon that resembled the European malting varieties in a number of traits.

\section{Acknowledgement}

The presented results were processed with the support of the Ministry of Agriculture of the Czech Republic, institutional support MZE-R01118 and research project QK1910197. The authors thank to the Agricultural Research Institute Kromeriz, Ltd. for providing the seed samples from The National Programme on Conservation and Utilization of Plant Genetic Resources and Agro-biodiversity.

\section{References}

Amabile, R.F., Faleiro, F.G., Capettini, F., Sayd, R.M., Peixoto, J.R., Guercia, R.F. (2014). Characterization and genetic variability of barley accessions (Hordeum vulgare L.) irrigated in the savannas based on malting quality traits. Journal of The Institute of Brewing, 120(4), 404-414. https://doi.org/10.1002/jib.179

Bockelman, H.E., Valkoun, J. (2010). Barley germplasm conservation and resources. In: Ullrich, S.E. (Ed.) Barley: improvement, production, and uses. Blackwell Publishing, Chapter 7, p. 144-159. ISBN 978-0-470-95862-9

Cativelli, L., Ceccarelli, S., Romagosa, I., Stanca, M., (2011). Abiotic Stresses in Barley: Problems and Solutions. In: Ullrich, S.E. (Ed.) Barley: improvement, production, and uses. Blackwell Publishing, Chapter 10, pp. 46. Online ISBN 9780470958636, https://doi. org/10.1002/9780470958636.ch10

Condón, F., Rasmusson, D.C., Schiefelbein, E., Velasquez G., Smith, K.P. (2009). Effect of advanced cycle breeding on genetic gain and phenotypic diversity in barley breeding germplasm. Crop Science, 49(5), 1751-1761. https://doi.org/10.2135/cropsci2008.10.0585 
EBC Analysis Committee (2010). Analytica EBC. Nürnberg, Fachverlag Hans Carl. ISBN 978-3-418-00759-5.

FAOSTAT (2017). Crop Statictis. In: fao.org [online]. [cit. 22. 7. 2020]. Available from: http://www.fao.org/faostat/en/?\#data/QC

Fox, G.P., Panozzo, J.F., Li, C.D., Lance, R.C.M., Inkerman, P.A., Henry, R.J. (2003). Molecular basis of barley quality. Australian Journal of Agricultural Research 54(12), 1081-1101. https://doi.org/10.1071/ AR02237

Fox, G.P., Kelly, A.M., Poulsen, D.M.E., Inkerman, P.A., Henry, R.J. (2006). Genetic and environmental effects on selecting improved barley grain size in dry environments. Journal of Cereal Science, 43(2), 198-208. https://doi.org/10.1016/j.jcs.2005.08.004

Hartman, I., Svobodová, I., Spáčilová, V., Míša, P. (2017). Reakce odrůd sladovnického ječmene na pěstování v režimu nízkých vstupů („low-input“) a ekologickém režimu II. Část Sladovnická kvalita. Obilnářské listy, 25(3-4), 90-93.

Jin, X., Cai, S., Han, Y., Wang, J., Wei, K., Zhang, G. (2011). Genetic variants of HvGlb1 in Tibetan annual wild barley and cultivated barley and their correlation with malt quality. Journal of Cereal Science, 53(1), 59-64. https://doi.org/10.1016/j.jcs.2010.09.006

Leistrumaitë, A., Paplauskienë, V. (2005). Genetic resources of spring barley: screening for yield stability and grain malt quality traits. Biologija, 3, 23-26.

Matthies, I.E., Malosetti, M., Röder, M.S., van Eeuwijk, F. (2014). Genome-Wide Association Mapping for Kernel and Malting Quality Traits Using Historical European Barley Records. PLoS ONE 9(11), e110046. https://doi.org/10.1371/journal.pone.0110046

MEBAK (2011). Raw Materials: Barley; Adjuncts; Malt; Hops and Hop Products; Collection of Brewing Analysis Methods of the Mitteleuropäische Brautechnische Analysenkommission. MEBAK, Freising-Weihenstephan, $341 \mathrm{pp}$.

Morgan, A.G., Riggs, T.J. (1981). Effect of Drought on Yield and on Grain and Malt Characters in Spring Barley. Journal of the Science Food and Agriculture 32(4), 339-346. https://doi.org/10.1002/ jsfa.2740320405
MZe (2017). National Program on Conservation and Utilization of Plant Genetic Resources and Agro-biodiversity. The Ministry of Agriculture of the Czech Republic, 51834/2017-MZE-17253.

Prokeš, J., Vaculová, K., Milotová, J. (2007). Selected malting parameters of new accessions from the collection of spring barley genetic resources. Kvasny Prumysl, 53(6), 162-167. https://doi.org/10.18832/ kp2007007

Przulj, N.V., Momcilovic, V., Crnobarac, J. (2013). Path coefficient analysis of quality of two-row spring barley. Genetika 45(1), 21-30. https:// doi.org/10.2298/GENSR1301021P

Przulj, N., Momcilovic, V., Simic, J., Mirosa Vljevic, M. (2014). Effect of growing season and variety on quality of spring two-rowed barley. Genetika, 46(1), 59-73. https://doi.org/10.2298/GENSR1401059P

Psota, V. (2008). Historical and current varieties of spring barley, varieties suitable for "České pivo". Kvasny Prumysl, 54(11-12), 326-331. https://doi.org/10.18832/kp2008017

Psota, V., Krajčovič, T., Križanová, K., Sachambula, L. (2017). History and progress of barley breeding in Sládkovičovo. Kvasny Prumysl, 63(2),70-80. https://doi.org/10.18832/kp201710

Psota, V., Dvořáčková, O., Nečas, M., Musilová, M. (2020). Barley and malt. In: Psota, V. (ed.): Barley Year Book 2020. Praha VÚPS. ISBN 978-8086576-91-6

Savin, R., Nicolas, M. E. (1996): Effects of Short Periods of Drought and High Temperature on Grain Growth and Starch Accumulation of Two Malting Barley Cultivars. Australian Journal of Plant Physiology, 23(2), 201-210. https://doi.org/10.1071/PP9960201

Savin, R., Nicolas, M. E. (1999). Effect of timing of heat stress and drought on growth and quality of barley grains. Australian Journal of Agricultural Research 50(3), 357-364. https://doi.org/10.1071/A98080

Šimić, G., Lalić, A., Horvat, I., Abičič, I., Berakovič, I. (2015). $\beta$-glucan content and $\beta$-glucanase activity of winter and spring malting barley cultivars. Acta Alimentaria, 44(4), 542-548. https://doi. org/10.1556/066.2015.44.0026

Zheng, X., Li, L., Wang, X. (2011). Molecular characterization of arabinoxylans from hull-less barley milling fractions. Molecules, 16(4), 27432753. https://doi.org/10.3390/molecules16042743 